\title{
Broadband Amplification of Low-Terahertz Signals Using Axis-Encircling Electrons in a Helically Corrugated Interaction Region
}

\author{
W. He, C. R. Donaldson, L. Zhang, K. Ronald, A. D. R. Phelps, and A. W. Cross \\ Department of Physics, SUPA, University of Strathclyde, Glasgow G4 ONG, United Kingdom \\ (Received 11 April 2017; published 31 October 2017)
}

\begin{abstract}
Experimental results are presented of a broadband, high power, gyrotron traveling wave amplifier (gyroTWA) operating in the (75-110)-GHz frequency band and based on a helically corrugated interaction region. The second harmonic cyclotron mode of a $55-\mathrm{keV}, 1.5-\mathrm{A}$, axis-encircling electron beam is used to resonantly interact with a traveling $\mathrm{TE}_{21}$-like eigenwave achieving broadband amplification. The gyroTWA demonstrates a $3-\mathrm{dB}$ gain bandwidth of at least $5.5 \mathrm{GHz}$ in the experimental measurement with $9 \mathrm{GHz}$ predicted for a wideband drive source with a measured unsaturated output power of $3.4 \mathrm{~kW}$ and gain of 36-38 dB. The approach may allow a gyro-TWA to operate at $1 \mathrm{THz}$.
\end{abstract}

DOI: 10.1103/PhysRevLett.119.184801

Generation of high power, high frequency radiation in the low-terahertz [(0.1-1)-THz] range has attracted great interest in the last few decades due to its potential applications in the areas of communications, remote sensing, plasma heating, and electron spin resonance (ESR) spectroscopy. The output power from conventional "slow wave" microwave sources, such as traveling wave tubes and klystrons, drops dramatically in this frequency range due to the small cross-sectional dimensions of their beam-wave interaction regions. The lack of tabletop broadband powerful $(\mathrm{kW})$ amplifiers in this frequency range is a pressing issue, forming part of the well-known "THz gap." By avoiding the use of a slow wave structure, gyrodevices based on the cyclotron resonance maser (CRM) instability, offer higher output power capability [1]. By operating in high order modes, gyrotrons have demonstrated high power (MW) [2] and terahertz operation [3-6]. However, they can only operate at discrete frequencies. Applications such as wireless communications, radar, and ESR require a large frequency bandwidth and high power. For example, to achieve large spin polarization of paramagnetic electrons, high-field pulsed ESR requires sophisticated short pulse sequences of millimeter-wave radiation of a few kilowatts in power with precise phase and amplitude control [7] which can only be provided by a broadband gyrotron traveling wave amplifier (gyro-TWA) [8].

The gyro-TWA with a smooth-bore interaction region has a hyperbolic wave dispersion curve and a linear beam line which lead to limited amplification bandwidth. To achieve a wide amplification bandwidth, such a gyro-TWA has to operate in a region with a large value of axial wave number

Published by the American Physical Society under the terms of the Creative Commons Attribution 4.0 International license. Further distribution of this work must maintain attribution to the author(s) and the published article's title, journal citation, and DOI. where the spread in the axial velocity acts to reduce the interaction efficiency and bandwidth due to the broadening of the electron cyclotron line. Smooth-bore gyro-TWA experiments in $K a$ band have achieved $70-\mathrm{dB}$ saturated gain, saturated power and gain bandwidths of $~ 9 \%$ and $\sim 3.8 \%$, respectively, with a maximum $93 \mathrm{~kW}$ of output power [9]. A number of scaled experiments in the $W$ band were reported which achieved a gain bandwidth of $\sim 1 \%$, for example, Song et al. achieved $\sim 1.3 \%$ gain bandwidth and high power of $59 \mathrm{~kW}$ with 42-dB saturated gain in 2004 [10] with the output power and gain improved in 2006 [11]. Blank et al. achieved an impressive 3-dB power bandwidth of $\sim 6.8 \%$ in 2007 , though the gain bandwidth is not explicitly quoted [12]. Yan et al. achieved $90-112 \mathrm{~kW}$ in an amplifier with a saturated power bandwidth of 93$97 \mathrm{GHz}$ and a 3-dB bandwidth of 1\% [13]. At yet higher frequencies, a bandwidth was reported at $250 \mathrm{GHz}$ of $1.8 \%$ with an output power of $4.4 \mathrm{~W}$ [14]. Both the saturated power bandwidth (implying variation in input power) and gain bandwidth (where this is characterized on the gain curve) are important characteristics of an amplifier.

In contrast, a gyro-TWA based on a helically corrugated interaction region (HCIR) achieves larger instantaneous bandwidth by exploiting an eigenwave with a favorable dispersion. The inner surface of the HCIR takes the form of

$$
r_{(\theta, z)}=r_{0}+r_{1} \cos \left(m_{B} \theta-2 \pi z / d\right),
$$

where $r_{0}$ is the mean radius of the circular waveguide, $r_{1}$ is the corrugation amplitude, $m_{B}$ is the fold number, and $d$ is the axial period of the corrugation. The azimuthal and axial periodicities allow the resonant coupling of two normal modes to generate new eigenwaves. For example, a threefold $\operatorname{HCIR}\left(m_{B}=3\right)$ allows the coupling of the $\mathrm{TE}_{21}$ mode and the first spatial harmonic of the $\mathrm{TE}_{11}$ mode. One of the resultant eigenwaves has nearly constant group velocity over a large frequency range at near zero axial wave number and can be matched to resonantly interact with a 


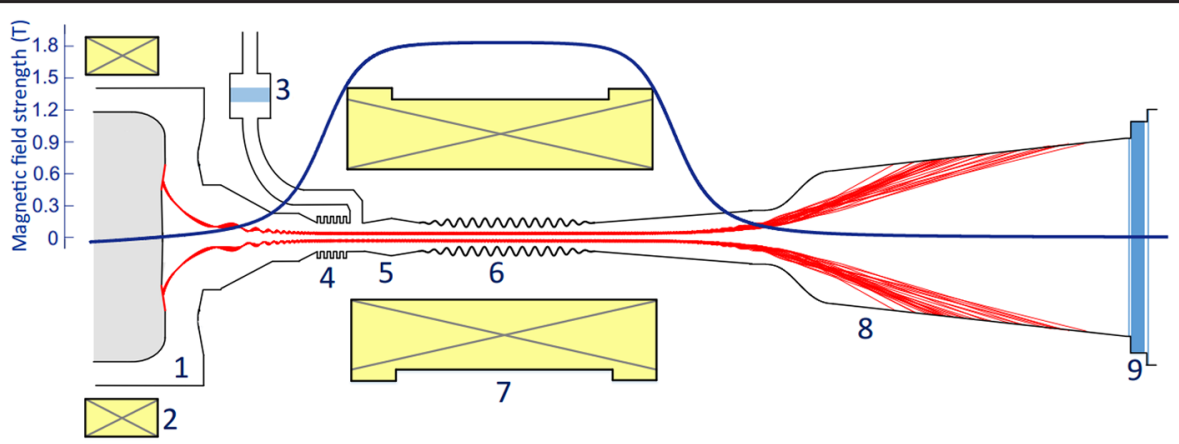

FIG. 1. The experimental setup of the gyro-TWA (1, cusp gun; 2, reverse coil; 3, pillbox window; 4, sidewall input coupler with broadband reflector; 5, linear-to-circular wave converter; 6, HCIR; 7, cavity coil; 8, smoothly profiled horn; 9, multiple-layer window).

cyclotron mode of the electron beam for broadband, highly efficient interaction, such as gyrotron backward wave oscillators $[15,16]$ and gyro-TWAs achieving an instantaneous gain bandwidth of over $20 \%[17,18]$. Theoretical study of a $W$-band gyro-TWA coupling the $\mathrm{TE}_{21}$ and $\mathrm{TE}_{31}$ modes on a fivefold HCIR has shown scope to achieve $6 \%$ bandwidth with an output power of $80 \mathrm{~kW}$ [19].

This Letter presents, for the first time, experimental measurements of a $W$-band [(75-110)-GHz] gyro-TWA using a threefold HCIR, with dimensions $r_{0}=1.45 \mathrm{~mm}$, $r_{1}=0.23 \mathrm{~mm}$, and $d=3.20 \mathrm{~mm}$, demonstrating high power and greatly increased gain bandwidth compared to any existing gyro-TWAs operating in the same frequency band. Zero-drive stable amplification was achieved generating a pure Gaussian output mode with a $-3-\mathrm{dB}$ gain bandwidth measured to be at least $5.5 \mathrm{GHz}$ (limited by the bandwidth of the seed signal) and an unsaturated output power of $3.4 \mathrm{~kW}$ corresponding to an electronic efficiency of $\sim 4 \%$. The output power of the gyro-TWA could reach $\sim 10 \mathrm{~kW}$ according to simulation if an input power of $3 \mathrm{~W}$ was available. Another key benefit of this approach is the ability to operate at the second harmonic using an axisencircling electron beam, thus, decreasing the required magnetic field by a factor of 2 . This factor of 2 is important at the $W$ band where the field is reduced from about $3.6 \mathrm{~T}$ to $1.8 \mathrm{~T}$. However, if the gyroamplifier is to be extended to $1 \mathrm{THz}$ in frequency, the required magnetic field will be $18 \mathrm{~T}$, which is feasible. It makes the demonstration of this technique very important and potentially crucial to the future development and applications of terahertz amplification.

A schematic drawing of the experimental setup is shown in Fig. 1. The millimeter-wave seed signal was coupled into the ultrahigh vacuum system through a broadband [(88-104)$\mathrm{GHz}$, high transmission $(-0.6 \mathrm{~dB})$ pillbox microwave window. A sidewall coupler with a $T$-junction structure was used to convert the $\mathrm{TE}_{10}$ mode in the rectangular waveguide into the $\mathrm{TE}_{11}$ mode in the circular waveguide. An optimized broadband Bragg reflector was used at one port of the $T$ junction to allow the electron beam to pass without interception and at the same time to minimize propagation of millimeter waves into the electron gun region. A polarizer was used after the millimeter-wave coupler to convert the linearly polarized $\mathrm{TE}_{11}$ wave into a circularly polarized one, to ensure all the drive power is in the correct polarization for excitation of the desired operating eigenwave in the HCIR. After the input millimeter wave was amplified in the interaction region, the generated high power radiation was converted into a Gaussian beam by using a smoothly profiled horn [20]. The horn was optimized to achieve a high Gaussian content (>98\%) with negligible reflection $(-37 \mathrm{~dB})$ over the operating bandwidth. The far-field radiation patterns of the output antenna were measured using a $W$-band vector network analyzer (VNA) (Anritsu ME7808) and showed good agreement with the simulated results. The wave was coupled out of the system through a multiple-layer window [21]. The reflection of the multiple-layer window was measured to be less than $-33 \mathrm{~dB}$ with negligible power absorption. The VNA was used to measure the millimeterwave properties, such as Ohmic loss and scattering parameters, of each individual component both separately and when assembled to form the overall gyro-TWA.

The gyro-TWA beam-wave dispersion and the small signal gain can be calculated using the following normalized equation [22]:

$$
\begin{aligned}
& {\left[\left(k_{z n}^{2}-2 \omega_{n}\right)\left(k_{z n}+\Delta_{g}-\omega_{n} / h_{0}\right)+2 \sigma^{2} / h_{0}\right]} \\
& \quad \times\left[k_{z n}-\left(\omega_{n}-\Delta_{H}\right) / \beta_{z 0}\right]^{2} \\
& =C^{3}\left(k_{z n}+\Delta_{g}-\omega_{n} / h_{0}\right) \\
& \quad \times\left\{1+\frac{2 s}{\alpha_{0}^{2} \beta_{z 0}}\left[k_{z n}-\left(\omega_{n}-\Delta_{H}\right) / \beta_{z 0}\right]\right\},
\end{aligned}
$$

while the definitions of the symbols in Eq. (2) can be found in Ref. [16], $\omega_{n}$ and $k_{z n}$ are the normalized frequency and axial wave number. The dispersion of the eigenwave and the electron beam could be calculated by solving the uncoupled beam-wave equation by setting the Pierce factor $C=0$ in Eq. (2). In a general case, Eq. (2) has four $\omega_{n}\left(k_{z n}\right)$ roots, with two real roots being the "hot" (electron beam present) eigenwaves, and a pair of conjugate complex roots, which are degenerates of the electron cyclotron mode due to the CRM interaction, when the beam parameters are suitably 


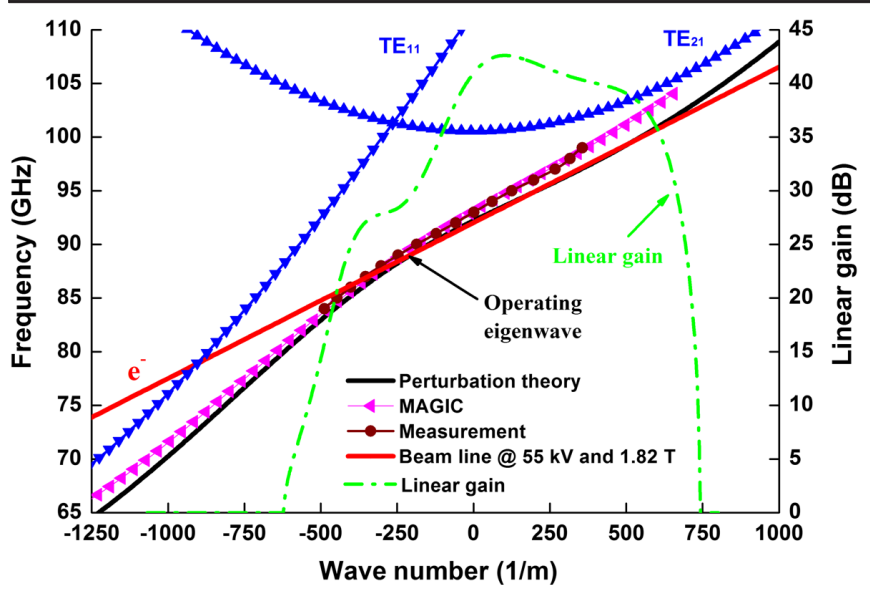

FIG. 2. The dispersion curve of the threefold helically corrugated waveguide and the beam line.

chosen. The negative imaginary number of the solution gives rise to the small signal gain of the interaction. The dispersion diagram of the first spatial harmonic of the $\mathrm{TE}_{11}$, the coupling $\mathrm{TE}_{21}$ mode, the operating eigenwave, and small signal gain were calculated and are shown in Fig. 2. The measured dispersion properties of the operating eigenwave in comparison to the results from numerical simulation using the $3 \mathrm{D}$ finite-difference time-domain particle-in-cell code MAGIC [23] are also shown in Fig. 2. The measured and simulated dispersions of the operating eigenwave were achieved by the methods described in Ref. [16].

The electron beam used to drive the beam-wave interaction was produced by a thermionic cusp electron gun [24]. After being emitted from the annular cathode, the electrons accelerated by the electric field also experience a reversal of magnetic field direction with the cusp point just a few millimeters downstream from the cathode surface. As a result of conservation of the canonical angular momentum, an axis-encircling electron beam was formed when the electric and magnetic fields were properly matched. The electron beam was then compressed to achieve the desired velocity alpha $\left(\alpha=v_{\perp} / v_{\|}\right)$of $\sim 1$ for beam-wave interaction. $\alpha$ could be tuned by changing the amplitude of the magnetic field at the cathode surface. The use of an axis-encircling electron beam is advantageous in suppressing mode competition for a harmonic gyrodevice, as such a beam only interacts with a waveguide mode $\mathrm{TE}_{m n}$ whose azimuthal index $m$ equals the harmonic number $s$ of the electron beam.

In the experiment, the magnetic field was $\sim 1.82 \mathrm{~T}$ when the beam voltage was $55 \mathrm{kV}$. The operating beam voltage $\alpha$ and the required magnetic field in the interaction region were chosen so that the beam line was matched with the dispersion curve of the operating eigenwave for broadband amplification.

The high voltage pulse to accelerate the electron beam was generated by a stacked double-Blumlein transmission line and a spark-gap switch. The rise time was about 30$35 \mathrm{~ns}$, and the pulse flat top was about $350 \mathrm{~ns}$. Diagnostics with a fast response time were used to measure the beam voltage, beam current, and microwave signal. Prior to the millimeter-wave experiment, the current and the crosssectional dimensions of the electron beam were measured separately by using a Faraday cup and a scintillator disk that were inserted into the beam tube at the end of the interaction region. The thermionic cathode was operated in the temperature-limited regime, and, therefore, the beam current could be controlled by varying the heating power of the cathode. The cross-sectional images of the electron beam were used to calculate $\alpha$.

The input millimeter-wave signal was from a tunable solid-state source with a maximum output power of 1.5$1.8 \mathrm{~W}$ in the (90-96.5)-GHz frequency range (Quinstar QAR-90A12319Z10). The output power level of the source can be programmed from 0 to its maximum value of $1.5 \mathrm{~W}$ and was calibrated using a $W$-band power meter. The output frequency of the source was calibrated using an inband fundamental mixer (Millitech MXP-10-R). Its local oscillator signal was produced by a Gunn diode (Millitech GDM-10-1013IR) at $94.9 \mathrm{GHz}$. The resultant intermediate frequency (IF) signal was directly measured by a $20-\mathrm{GHz}$ digitizing oscilloscope (Agilent DSX-X 92004A).

The amplified high power microwave pulse of the gyroTWA radiated out through the multiple-layer window was measured by a crystal detector located in a shielded metal box and positioned $80 \mathrm{~cm}$ away from the millimeter-wave window. A standard gain $W$-band pyramidal horn and a calibrated variable 0 - to $90-\mathrm{dB}$ rotary vane attenuator were used before the detector so that the millimeter radiation before and after amplification could be measured. To find the amplification regime of the gyro-TWA, the magnitudes of the magnetic fields at the cavity and cathode were systematically scanned. The amplifier was found to be "zero-drive" stable when the electron beam had an $\alpha$ of $\sim 1$ and the cavity magnetic field was $\sim 1.82 \mathrm{~T}$. Typical time-correlated traces of the beam voltage, beam current, and the amplified millimeter-wave signal were recorded and are shown in Fig. 3.

The output frequency of the gyro-TWA was also measured by the heterodyne mixing method. When the input frequency was set at $93 \mathrm{GHz}$, the mixer signal was recorded by the oscilloscope and is also shown in Fig. 3. The spectral analysis showed a clear peak IF amplitude at $1.9 \mathrm{GHz}$, which proved the amplification of the input signal.

The responses of the crystal detector at different input power levels and different separations from the output window of the gyro-TWA were carefully calibrated. From the detector output in the "cold" and "hot" (without and with electron beam amplification) experimental conditions, the gain and the output power of the gyro-TWA at different input signal levels were measured. The whole transmission loss of the system was carefully measured by the VNA to be $\sim 5-6 \mathrm{~dB}$, which meant the maximum cold power without amplification was $\sim 0.52 \mathrm{~W}$. Figure 4 shows the measured gain and output power at $93 \mathrm{GHz}$ as a function of 

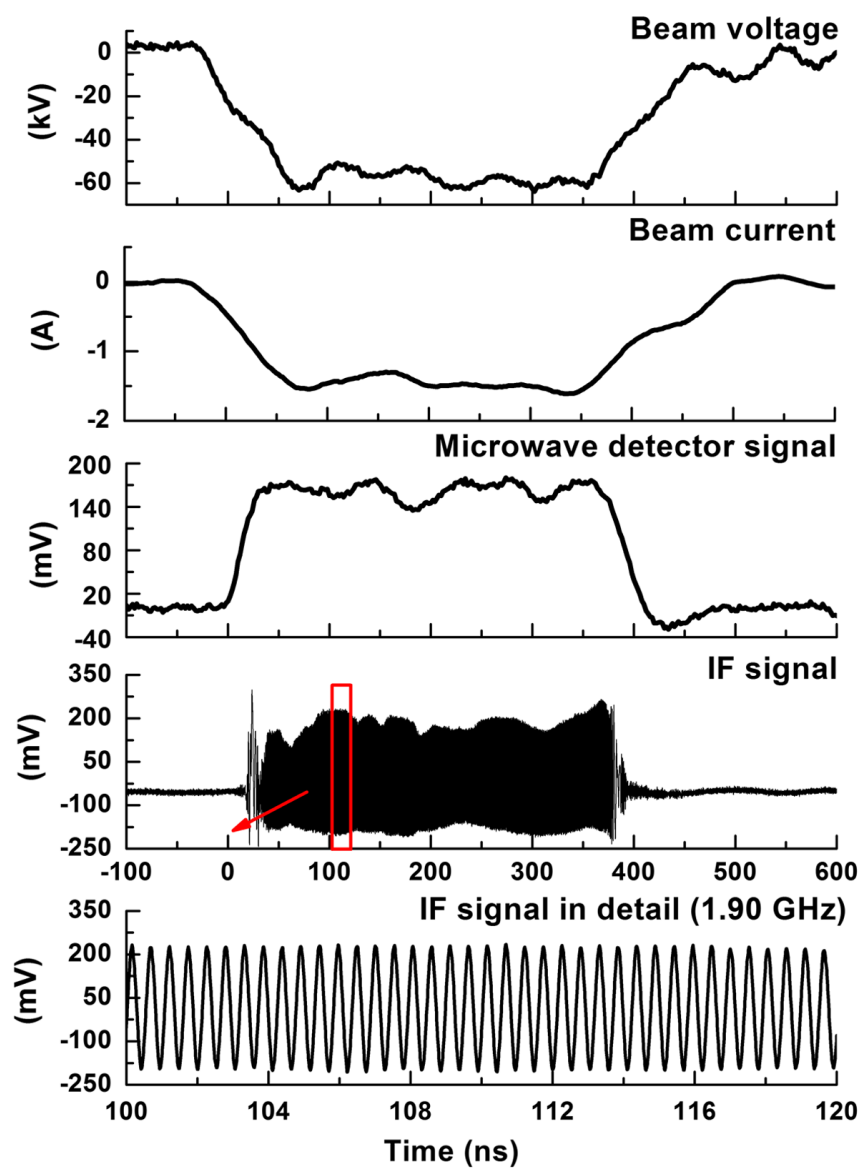

FIG. 3. Measured time-correlated beam voltage, current, millimeter-wave signal, and the IF signal from mixer.

input power. The gain was measured to be $\sim 40-43 \mathrm{~dB}$ when the cold signal was in the range of $0.02-0.15 \mathrm{~W}$. With the increase of the power of the seed signal, the gain reduced slightly to about $38 \mathrm{~dB}$ at $0.5 \mathrm{~W}$ input power. Figure 4 shows that the amplifier had not reached saturation, the maximum output power being limited by the input power. The output power and amplification of the gyro-TWA as a function of input power at $93 \mathrm{GHz}$ using beam parameters consistent with the experiment were simulated using the particle-in-cell code MAGIC. A righthand, annular, axis-encircling electron beam with a thickness of $0.1 \mathrm{~mm}$ and $\alpha$ of 1 was used to interact with a circularly polarized wave in the simulation. The simulated output power and gain were in excellent agreement with the measured results as the input power ranged from 0.01 to $0.52 \mathrm{~W}$ if an $\alpha$ spread of $10 \%$ is assumed. The results of the simulation are also shown in Fig. 4. The saturated output power of $10 \mathrm{~kW}$ could be realized with a more powerful input source.

The cold power transmitted through the amplifier was measured as a function of the frequency and is shown in Fig. 5. The power of the cold signal was negligible when the frequency was above $96.5 \mathrm{GHz}$. The bandwidth of the gyro-TWA at the maximum available input power was also

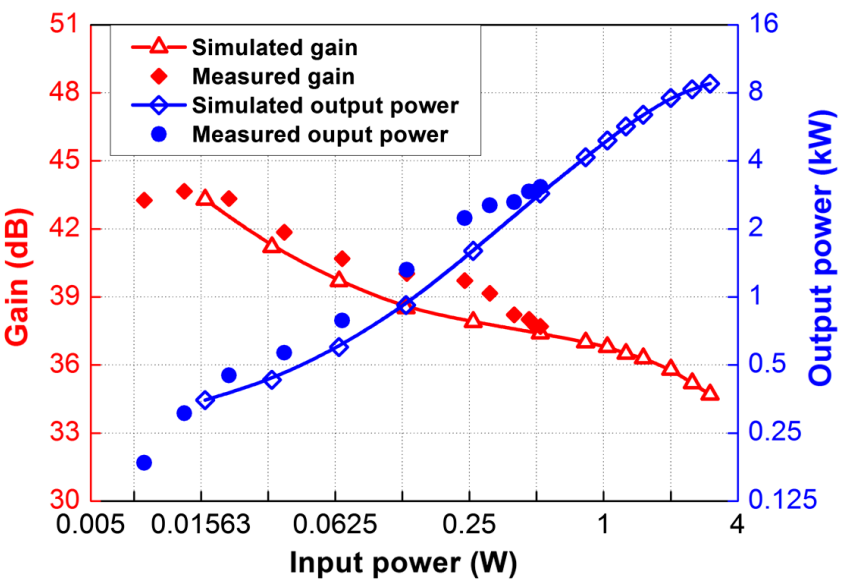

FIG. 4. The measured and simulated gain and output power at different input power levels at $93 \mathrm{GHz}$.

measured, and the result is shown in Fig. 5. The peak output power of about $3.4 \mathrm{~kW}$ was measured at $94 \mathrm{GHz}$. However, it was not possible to determine the gain of the gyro-TWA above $96.5 \mathrm{GHz}$ in the same conditions due to the input source being unable to generate significant millimeterwave radiation at these frequencies. The simulated gain bandwidth assuming an $\alpha$ spread of $10 \%$ was found to be in good agreement with the measured results and is also shown in Fig. 5.

The far-field radiation pattern of the output millimeter waves generated by the gyro-TWA was measured to verify the Gaussian mode content. The simulated and measured results of the radial $E$-field component in the $E$ plane at $95 \mathrm{GHz}$ are shown in Fig. 6. The simulation was carried out using CST MicrowAVE STUDio. Taking into account a $2^{\circ}$ systematic error in the radial angle, the simulation and measurement results agreed well.

In the $W$-band gyro-TWA experiment, stable amplification over the frequency range of $91-96.5 \mathrm{GHz}$ was achieved from an electron beam of $55 \mathrm{kV}, 1.5 \mathrm{~A}$, and $\alpha$ of $\sim 1$. The maximum gain was measured to be $38 \mathrm{~dB}$ at $94 \mathrm{GHz}$ with a maximum input power of $0.52 \mathrm{~W}$, which

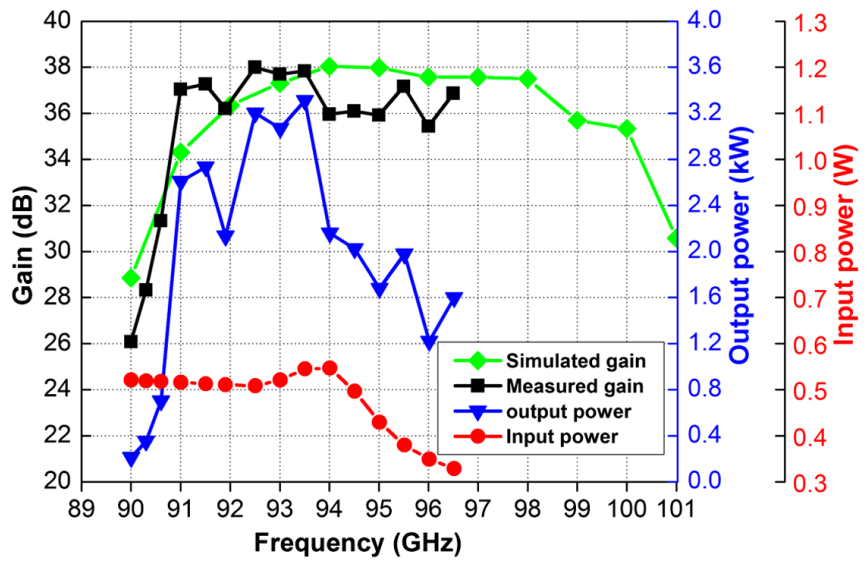

FIG. 5. The gain and output power at different frequencies. 


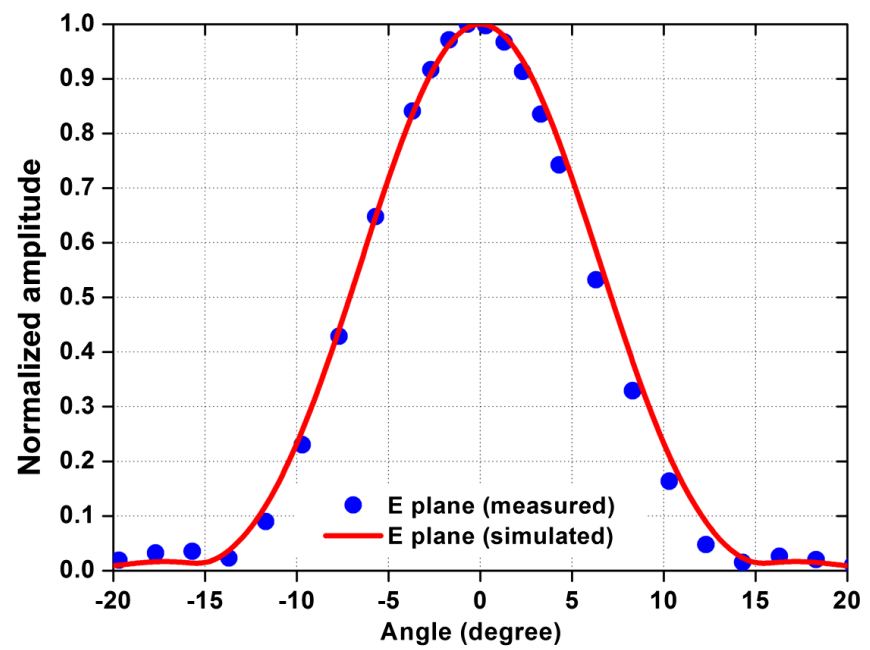

FIG. 6. The far-field measurement of the gyro-TWA.

gives a maximum output power of $3.4 \mathrm{~kW}$. The performance at higher input power levels and frequencies above $96.5 \mathrm{GHz}$ were not measured due to limitations of the input drive source. The results were in good agreement with a simulated maximum output power of $10 \mathrm{~kW}$ and bandwidth of 91-100 GHz. Therefore, the simulation code has shown that it could accurately predict the output power for gyrodevices based on HCIRs operating both in $X$ and $W$ band $[15,16]$. The simulation results from the same code indicate this experiment could be scaled up to operate at higher frequencies. A gyro-TWA operating at the central frequency of $263 \mathrm{GHz}$ with an output power of $\sim 1 \mathrm{~kW}$ and bandwidth of $5 \%$ is currently being studied at the University of Strathclyde.

The authors would like to thank the UK research councils EPSRC (research Grants No. EP/G036659/1 and No. EP/K029746/1) and STFC (research Grants No. ST/K006673/1 and No. ST/N002326/1) for supporting this work. Dr. Peter Huggard and Mr. Mat Beardsley of the Millimetre Wave Technology Group at the STFC Rutherford Appleton Laboratory, UK for the construction of the HCIR.

[1] K. R. Chu, Rev. Mod. Phys. 76, 489 (2004).

[2] J. Jelonnek et al., IEEE Trans. Plasma Sci. 42, 1135 (2014).

[3] V. L. Bratman, Y. K. Kalynov, and V. N. Manuilov, Phys. Rev. Lett. 102, 245101 (2009).

[4] M. Einat, M. Pilossof, R. Ben-Moshe, H. Hirshbein, and D. Borodin, Phys. Rev. Lett. 109, 185101 (2012).

[5] N. S. Ginzburg, I. V. Zotova, A. S. Sergeev, V. Y. Zaslavsky, and I. V. Zheleznov, Phys. Rev. Lett. 108, 105101 (2012).
[6] M. Y. Glyavin, A. G. Luchinin, and G. Y. Golubiatnikov, Phys. Rev. Lett. 100, 015101 (2008).

[7] D. R. Bolton, P. A. S. Cruickshank, D. A. Robertson, and G. M. Smith, Electron. Lett. 43, 346 (2007).

[8] H. J. Kim, E. A. Nanni, M. A. Shapiro, J. R. Sirigiri, P. P. Woskov, and R. J. Temkin, Phys. Rev. Lett. 105, 135101 (2010).

[9] K. R. Chu, H. Y. Chen, C. L. Hung, T. H. Chang, L. R. Barnett, S. H. Chen, and T. T. Yang, Phys. Rev. Lett. 81, 4760 (1998).

[10] H. H. Song, D. B. McDermott, Y. Hirata, L. R. Barnett, C. W. Domier, H. L. Hsu, T. H. Chang, W. C. Tsai, K. R. Chu, and N. C. Luhmann, Phys. Plasmas 11, 2935 (2004).

[11] L. R. Barnett, W. C. Tsai, H. L. Hsu, N. C. Luhmann, Jr., C. C. Chiu, K. F. Pao, and K. R. Chu, in 2006 IEEE International Vacuum Electronics Conference held Jointly with 2006 IEEE International Vacuum Electron Sources (IEEE, New York, 2006), p-. 461-462.

[12] M. Blank, P. Borchard, S. Cauffman and K. Felch, in 2007 Joint 32nd International Conference on Infrared and Millimeter Waves and the 15th International Conference on Terahertz Electronics (IEEE, New York, 2007), pp. 364-366.

[13] R. Yan, Y. Tang, and Y. Luo, IEEE Trans. Electron Devices 61, 2564 (2014).

[14] E. A. Nanni, S. M. Lewis, M. A. Shapiro, R. G. Griffen, and R. J. Temkin, Phys. Rev. Lett. 111, 235101 (2013).

[15] W. He, A. W. Cross, A. D. R. Phelps, K. Ronald, C. G. Whyte, S. V. Samsonov, V. L. Bratman, and G. G. Denisov, Appl. Phys. Lett. 89, 091504 (2006).

[16] W. He, C. R. Donaldson, L. Zhang, K. Ronald, P. McElhinney, and A. W. Cross, Phys. Rev. Lett. 110, 165101 (2013).

[17] V. L. Bratman, A. W. Cross, G. G. Denisov, W. He, A. D. R. Phelps, K. Ronald, S. V. Samsonov, C. G. Whyte, and A. R. Young, Phys. Rev. Lett. 84, 2746 (2000).

[18] S. V. Samsonov et al., IEEE Trans. Electron Devices 61, 4264 (2014).

[19] S. V. Mishakin, S. V. Samsonov, and G. G. Denisov, IEEE Trans. Electron Devices 62, 3387 (2015).

[20] L. Zhang, W. He, C. R. Donaldson, G. M. Smith, D. A. Robertson, R. I. Hunter, and A. W. Cross, IEEE Trans. Electron Devices 64, 2665 (2017).

[21] C. R. Donaldson, P. McElhinney, L. Zhang, and W. He, IEEE Trans. Terahertz Sci. Technol. 6, 108 (2016).

[22] G. G. Denisov, V. L. Bratman, A. D. R. Phelps, and S. V. Samsonov, IEEE Trans. Plasma Sci. 26, 508 (1998).

[23] T. Gray, D. N. Smithe, and L. D. Ludeking, Introduction to MAGIC (Mission Research Corporation, Newington, VA, 2003).

[24] C. R. Donaldson, W. He, A. W. Cross, F. Li, A. D. R. Phelps, L. Zhang, K. Ronald, C. W. Robertson, C. G. Whyte, and A. R. Young, Appl. Phys. Lett. 96, 141501 (2010). 